TRANSACTIONS OF THE

AMERICAN MATHEMATICAL SOCIETY

Volume 352 Number 7 , Pages 3289-3303

S 0002-9947(00)02607-6

Article electronically published on March 24, 2000

\title{
ABSOLUTELY CONTINUOUS S.R.B. MEASURES FOR RANDOM LASOTA-YORKE MAPS
}

\author{
JÉRÔME BUZZI
}

\begin{abstract}
A. Lasota and J. A. Yorke proved that a piecewise expanding interval map admits finitely many ergodic absolutely continuous invariant probability measures. We generalize this to the random composition of such maps under conditions which are natural and less restrictive than those previously studied by Morita and Pelikan. For instance our conditions are satisfied in the case of arbitrary random $\beta$-transformations, i.e., $x \mapsto \beta x \bmod 1$ on $[0,1]$ where $\beta$ is chosen according to any stationary stochastic process (in particular, not necessarily i.i.d.) with values in $] 1, \infty[$.

RÉsumé. A. Lasota et J. A. Yorke ont montré qu'une application de l'intervalle dilatante par morceaux admet un nombre fini de mesures de probabilité invariantes et ergodiques absolument continues. Nous généralisons ce résultat à la composition aléatoire de telles applications sous des conditions naturelles, moins restrictives que celles précédemment envisagées par Morita et Pelikan. Par exemple, nos conditions sont satisfaites par toute $\beta$-transformation aléatoire, i.e., $x \mapsto \beta x \bmod 1$ sur $[0,1]$ avec $\beta$ choisi selon un processus stochastique stationnaire quelconque (en particulier, non-nécessairement i.i.d.) à valeurs dans $] 1, \infty[$.
\end{abstract}

\section{INTRODUCTION}

0.1. Setting. We are interested in the "chaotic" dynamics defined by maps of the following, well-known type:

Definition 0.1. $f:[0,1] \rightarrow[0,1]$ is a Lasota-Yorke map if there exists a finite subdivision $a_{0}=0<a_{1}<\cdots<a_{N}=1$ of the unit interval such that, for each restriction $f_{i}$ of $f$ to any subinterval $] a_{i-1}, a_{i}[, i=1, \ldots, N$ :

1. $f_{i}$ is a homeomorphism on its image;

2. $f_{i}$ is non-singular with respect to Lebesgue measure.

We shall assume that $N$ is minimal. We call the intervals $] a_{i-1}, a_{i}[$ the intervals of $f$, and define $N(f)=N, \delta(f)=\inf \left\{\left|f^{\prime}(x)\right|: x \in[0,1] \backslash\left\{a_{0}, \ldots, a_{N}\right\}\right\}$ and $\operatorname{var}\left(f^{\prime}\right)=\sup _{a} \sum_{i=1}^{m}\left|f^{\prime}\left(a_{i}\right)-f^{\prime}\left(a_{i-1}\right)\right|, a=\left(a_{0}=0<a_{1}<\cdots<a_{m}=1\right)$, ranging over the finite subdivisions of $[0,1]$, which is the variation of $\left|f^{\prime}\right|$, a fixed jacobian of $f$.

By a well-known result of A. Lasota and J. A. Yorke 19, the iteration of any map of this type is "chaotic" whenever the map is expanding (i.e., $\delta(f)>1$ ). By

Received by the editors Febrary 25, 1998.

2000 Mathematics Subject Classification. Primary 37A25, 37H99.

Key words and phrases. Absolutely continuous invariant measures; piecewise monotonic maps; random maps; transfer operator; bounded variation.

Work partly done at the Laboratoire de Topologie de Dijon, Université de Bourgogne, France. 
"chaotic" one means that finitely many absolutely continuous invariant probability measures describe Lebesgue almost all orbits.

On the other hand, in higher dimensions, while everyone seems to think that the analoguous theorem is true, the currently known methods need a sufficient amount of expansion to kill the possible accumulation of singularities [12], or need to be restricted to generic situations [6], [9] where this accumulation does not take place (see however [8], 23] and, perhaps more surprisingly, 24]).

In this paper we consider an "intermediate case" of dimension " $1+\epsilon$ ": the random composition of Lasota-Yorke maps. One can define a random dynamical system $f$ on a space $M$ to be a stationary stochastic process $f_{1}, f_{2}, \cdots: M \rightarrow M$ (i.e., with values in a space of maps) (see for instance [15]). The associated random orbits are the stochastic processes

$$
x_{0}, x_{1}, \ldots \text { where } x_{0} \in X \text { and } x_{n+1}=f_{n+1}\left(x_{n}\right) .
$$

One can then ask about the asymptotic behavior of these random orbits. Observe that in general there is no measure simultaneously invariant under all the maps $f(\omega), \omega \in \Omega$. One considers instead S.R.B. measures (Sinai-Ruelle-Bowen measures), defined below, and one asks whether Lebesgue almost all orbits are described by "chaotic", i.e., absolutely continuous, S.R.B. measures.

We are going to prove that it is indeed the case in this " $1+\epsilon$-dimensional" Lasota-Yorke problem, as in the classical case.

0.2. Results. The stationary process $f_{1}, f_{2}, \ldots$ can be seen as given by $f_{n+1}=$ $f \circ T^{n}$, where $f$ is a measurable map defined on some abstract probability space $(\Omega, \mathcal{A}, \mathbb{P})$ and $T$ an automorphism. Write $\delta_{x}$ for the Dirac measure at a point $x, f_{\omega}$ for $f(\omega), \delta(\omega)$ for $\delta(f(\omega))$, etc. Then:

Definition 0.2. A probability measure $\mu$ on $M$ is S.R.B. for the random dynamical system $(\Omega, \mathcal{A}, \mathbb{P}, T, f)$ (or simply $f$ ) if, for $\mathbb{P}$-almost all $\omega \in \Omega$, the set $B_{\omega}(\mu)$ of points $x \in M$ such that

$$
\frac{1}{n} \sum_{k=0}^{n-1} \delta_{f_{T^{k-1} \omega^{\circ}} \cdots \circ f_{\omega}(x)} \stackrel{n \rightarrow \infty}{\longrightarrow} \mu \quad \text { vaguely }
$$

has positive Lebesgue measure. We call $B_{\omega}(\mu)$ the (random) basin of $\mu$.

In the case where $f_{\omega}$ is independent of $\omega$ this reduces to one of the classical definitions of an S.R.B. measure for a deterministic map.

Assumptions. A random Lasota-Yorke map $f$ is admissible if the following conditions are satisfied:

$$
\begin{gathered}
\left.\lim _{K \rightarrow \infty} \int_{\Omega} \log \delta(\omega) \wedge K d \mathbb{P}>0 \quad \text { (possibly }+\infty\right), \\
\log ^{+} \frac{N(\omega)}{\delta(\omega)} \text { is } \mathbb{P} \text {-integrable, } \\
\log ^{+} \operatorname{var}\left(1 /\left|f_{\omega}^{\prime}\right|\right) \text { is } \mathbb{P} \text {-integrable. }
\end{gathered}
$$

$(x \wedge y \stackrel{\text { def }}{=} \min (x, y))$.

We also assume $(\Omega, \mathcal{A}, \mathbb{P}, T)$ to be ergodic (otherwise one has to require (A1) for almost all ergodic components). 
Observe that we do not assume any kind of uniformity with respect to $\omega$, except the one which results from the fact that we are looking through an invariant measure $\mathbb{P}$. It should be noted in this connection that our results do not follow from analoguous statements about arbitrary sequences of maps. Indeed, there are sequences of maps $f_{1}, f_{2}, \ldots$ such that $\delta\left(f_{n}\right) \geq \delta_{*}, N\left(f_{n}\right) / \delta\left(f_{n}\right) \leq K$ and $\operatorname{var}\left(1 /\left|f_{n}^{\prime}\right|\right) \leq K$ for some $\delta_{*}>1$ and $K<\infty$, and nevertheless, $\left(f_{n} \circ \cdots \circ f_{1}\right)_{*} m$ converges to a singular measure (e.g., a Dirac measure). This is in contrast to settings like [2].

Moreover, our conditions are natural in the sense that if we suppress any one of the three conditions (A1), (A2) or (A3) then we can find even deterministic counter-examples to the existence of absolutely continuous invariant measures (this is easy for (A1) or (A2); see [13] for (A3)).

As usual, one associates to the random map $f$ the skew-product:

$$
\begin{aligned}
F: \Omega \times[0,1] & \longrightarrow \Omega \times[0,1] \\
(\omega, x) & \longmapsto\left(T \omega, f_{\omega} x .\right.
\end{aligned}
$$

Theorem 0.3. Let $(\Omega, \mathcal{A}, \mathbb{P}, T, f)$ be an admissible random Lasota-Yorke map.

Then the set of $F$-invariant probability measures absolutely continuous with respect to $\mathbb{P} \times m$ ( $m$ being Lebesgue measure), or, for short, a.c.i.m.'s, is a nonempty finite-dimensional simplex, generated by its ergodic elements.

Moreover the density of each such measure $\mu$ satisfies (after modification on a $\mathbb{P} \times m$-null set) the condition

$$
x \mapsto \frac{d \mu}{d \mathbb{P} \times m}(\omega, x) \text { has bounded variation for all } \omega \in \Omega,
$$

and we have strong convergence to equilibrium in the sense that, for each probability measure $\nu$ absolutely continuous with respect to $\mathbb{P} \times m$,

$$
\frac{1}{n} \sum_{k=0}^{n-1} \frac{d T^{k}(\nu)}{d \mathbb{P} \times m} \stackrel{n \rightarrow \infty}{\longrightarrow} \frac{d \mu}{d \mathbb{P} \times m} \quad \text { in } L^{1}(\mathbb{P} \times m),
$$

where $\mu$ is an a.c.i.m.

Finally, the number $r$ of ergodic a.c.i.m.'s is explicitly bounded by the non-trivial condition

$$
\int_{\Omega} \log \frac{1+(N(\omega)-1) / r}{\delta(\omega)} d \mathbb{P} \geq 0
$$

We formulate a probabilistic corollary of this.

Theorem 0.5. Any admissible random Lasota-Yorke map admits a finite number of S.R.B. measures, bounded by (0.4).

Moreover, the following hold:

1. These finitely many measures together describe almost all orbits in the sense that the union of their random basins has $\mathbb{P}$-almost surely total Lebesgue measure in $[0,1]$.

2. These S.R.B. measures are absolutely continuous.

0.3. Discussion of the results. Absolutely continuous S.R.B. measures for random maps have been mostly considered with respect to the related, but different problem of small random perturbations of a given deterministic dynamical system (see [16], 3] and the references there) or in the case of expanding mappings without singularities (see [15], [17], [5], [14], [1] and the references there). Random Lasota-Yorke maps have been considered by S. Pelikan [21] and T. Morita [20]. 
However, our conditions on the maps are much less restrictive then the ones in these two articles: in both papers the maps $f_{1}, f_{2}, \ldots$ are given by an i.i.d. process and in [20] the lengths of the intervals of the map are not too small whereas in 21] the maps must even be chosen from a finite set (we note that this last paper includes some situations outside the random composition of maps, as the probability of picking a given map is allowed to depend on the current position in $[0,1])$.

In particular, one of the motivations of this work was to be able to treat the following example, which could not be done by 21], 20.

0.4. Example. $f(x)=\beta x(\bmod 1)$, where $\beta_{1}, \beta_{2}, \ldots$ is an arbitrary stationary and ergodic stochastic process with values in $] 1, \infty[$.

This example turns out to be important for some applications (see [6]); for instance, it allows the reduction of the higher dimensional Lasota-Yorke problem to the one restricted to maps with constant Jacobian.

In this example, every critical point $c$ satisfies $f(c+)=f(0)=0$. Hence, it is easy to see (cf. section 3 ) that there is at most one a.c.i.m.

We also remark that the natural symbolic dynamics defined by our random maps are random subshifts which are not of finite type, contrary to the case of random smooth expanding maps investigated by T. Bogenschütz and V.M. Gundlach [4], [5].

0.5. Outline of proof. In the first section, we give two Lasota-Yorke inequalities estimating the variation of the density after the application of one given Lasota-Yorke map: one inequality, due to M. Rychlik, is well-known and gives a fine control over the variation provided that the intervals of the map are not too small; the other one is very crude but does not depend on the size of these intervals. Then, by applying these inequalities (the first one most of the time, the second one only when some interval of the map is very small), we prove that, for $h \in \mathcal{H}$, a dense subset of $L^{1}(\mathbb{P} \times m)$, the functions $\frac{1}{n} \sum_{k=0}^{n-1} \mathcal{L}^{k} h$ for $n=1,2, \ldots$ have their variation on each fiber $\omega \times[0,1]$ uniformly bounded with respect to $n$.

The second section builds an a.c.i.m. for the skew-product $F$ as an integrable function invariant under the transfer operator $\mathcal{L}$ defined by $F$ and $\mathbb{P} \times m$ :

$$
(\mathcal{L} h)(\omega, x)=\sum_{(\alpha, y) \in F^{-1}(\omega, x)} \frac{1}{\left|f_{\alpha}^{\prime}(y)\right|} h(\alpha, y)
$$

$\mathcal{L}$ satisfies the fundamental property $\int h \cdot \mathcal{L} g d \mathbb{P} \times m=\int h \circ F \cdot g d \mathbb{P} \times m$ for every $h \in L^{\infty}(\mathbb{P} \times m)$ and every $g \in L^{1}(\mathbb{P} \times m)$. Recall that $\mathbb{P}$ is preserved by $T$.

From the results of the first section, we deduce that the sequence $\frac{1}{n} \sum_{k=0}^{n-1} \mathcal{L}^{k} h$, $n \geq 1$, is weakly compact in $L^{1}(\mathbb{P} \times m)$ for each $h$ in a dense subset $\mathcal{H}$. We can therefore apply the Mean Ergodic Theorem and conclude the proof of existence.

Remark 0.6. The transfer operator $\mathcal{L}$ is not even bounded in $L^{\infty}(\mathbb{P} \times m)$, as we do not assume uniformity of expansion in (A1). Thus we cannot apply the IonescuTulcea-Marinescu Theorem as it is usually done.

In the third section, the explicit bound on the multiplicity of the a.c.i.m. will be deduced from the following geometric considerations. Because of the bounded variation property, the support of every a.c.i.m. must contain intervals in almost every fiber $\omega \times[0,1]$. Now the image of an interval has its length increased unless it contains some discontinuity. Working from this remark, one can see that every 
a.c.i.m. must "use" a certain number of the discontinuities. The bound then follows from assumption (A2). Finally we remark that finiteness can be proven much more abstractly.

We conclude by deducing from the theorem about the skew-product (Theorem 0.3) its probabilistic corollary (Theorem 0.5).

0.6. Perspectives. 1. Except for the explicit bound on the number of S.R.B. measures, the results and proofs presented in this paper only depend on having good Lasota-Yorke inequalities for a reasonable notion of variation. Hence they can be readily generalized, in particular, to some multi-dimensional settings. See [7.

2. One would like to know the ergodic and statistical properties of the S.R.B. measures constructed here. Under an additional and non-trivial assumption of strong topological mixing ("random covering"), we have proved [7] that there is indeed exponential decay of random correlations like in smooth settings [1], [2], [5], [14, [17. The proof is by Birkhoff cones. But this more precise result does not cover all the maps studied here and leaves open the (exciting if unlikely) possibility that some of those random maps, despite their uniform expansion, would have weak ergodic properties.

3. Are the S.R.B. measures constructed here stable with respect to (suitably defined) small perturbations?

4. Under what hypothesis would one be able to make statements (e.g., quasicompactness) about a global tranfer operator and therefore hope for much more precise results like those of [1].

Acknowledgements. I owe many thanks to Bernard Schmitt for stimulating discussions, as well as to Viviane Baladi, Gerhard Keller, Véronique Maume and Philippe Thieullen for useful comments.

\section{BOUNDS ON VARIATION}

This section establishes fiberwise bounds on the variation of $\mathcal{L}^{n} h$ for good functions $h$.

1.1. One-dimensional bounds. We can write $(\mathcal{L} h)(\omega, \cdot)=L_{f\left(T^{-1} \omega\right)} h\left(T^{-1} \omega, \cdot\right)$, where $L_{f}$ is the transfer operator associated to the interval map $f$ :

$$
L_{f} h(x)=\sum_{y \in f^{-1}(x)} \frac{1}{\left|f_{\omega}^{\prime}(y)\right|} h(y) \quad \text { for } h:[0,1] \rightarrow \mathbb{R}
$$

(recall that $T$ is an automorphism).

The basic ingredient is, as in the case of iteration of a fixed map, the following well-known inequality, proved by Rychlik (extending the inequality proved by Lasota and Yorke [19] for maps piecewise $C^{2}$ to absolutely continuous maps with bounded variation of the derivative).

Lemma 1.1 (M. Rychlik [22]). Let $f:[0,1] \rightarrow[0,1]$ be a Lasota-Yorke map with $\operatorname{var}\left(1 /\left|f^{\prime}\right|\right)<\infty$. Let $h:[0,1] \rightarrow \mathbb{R}$ be a function of bounded variation. Then

$$
\operatorname{var}\left(L_{f} h\right) \leq \frac{3}{\delta(f)} \operatorname{var}(h)+\beta(f)\|h\|_{1},
$$

where $\beta(f)<\infty$ depends measurably on $f$. 
A key feature of this estimate is the factor of 3 in front of the first term. It could be replaced by any number greater than 2 but not by 1 : this is related to the possibility, if $1<\delta(f)<2$, to have some interval mapped into itself. A recent paper of M. Blank and G. Keller [3] shows that this can produce unexpected behavior for random perturbations of some Lasota-Yorke maps in the limit when the size of the perturbation goes to zero.

In our setting, however, this factor of 3 turns out to be only a technical problem. We shall overcome it as in the case of the iteration of a fixed map by replacing our map with some high order iterate. However, in contrast to this classical case, the factor in front of the second term may then explode: $\beta(f \circ g)$ is not bounded by a function of $\beta(f)$ and $\beta(g)$. To control this we shall use the following more robust estimate:

Lemma 1.2. Let $f:[0,1] \rightarrow[0,1]$ be a Lasota-Yorke map. Let $h:[0,1] \rightarrow \mathbb{R}$ be a function of bounded variation. Then

$$
\operatorname{var}\left(L_{f} h\right) \leq\left[\frac{2 N(f)}{\delta(f)}+\operatorname{var}\left(1 /\left|f^{\prime}\right|\right)+\frac{1}{\delta(f)}\right] \operatorname{var}(h)+\left[\frac{2 N(f)}{\delta(f)}+\operatorname{var}\left(1 /\left|f^{\prime}\right|\right)\right]\|h\|_{1} .
$$

Proof. We have

$$
\begin{aligned}
\operatorname{var}\left(L_{f} h\right) \leq & \sum_{i=1}^{N} \operatorname{var}\left(\frac{h}{f^{\prime}} \circ f_{i}^{-1} \cdot 1_{f(] a_{i-1}, a_{i}[)}\right) \\
\leq & \sum_{i=1}^{N}\left|\frac{h}{f^{\prime}}\left(a_{i-1}\right)\right|+\left|\frac{h}{f^{\prime}}\left(a_{i}\right)\right|+\max \left(\frac{1}{\left|f^{\prime}\right|}\right) \operatorname{var}_{a_{a_{i-1}, a_{i}[}(h)} \\
& \quad+\operatorname{var}_{a_{i-1}, a_{i}[}\left(\frac{1}{\left|f^{\prime}\right|}\right) \max (|h|)
\end{aligned}
$$

using the bound $\operatorname{var}(f g) \leq \operatorname{var}(f) \max (|g|)+\max (|f|) \operatorname{var}(g)$. Hence,

$$
\begin{aligned}
\operatorname{var}\left(L_{f} h\right) & \leq\left[\frac{2 N(f)}{\delta(f)}+\operatorname{var}\left(\frac{1}{\left|f^{\prime}\right|}\right)\right] \max (|h|)+\frac{1}{\delta(f)} \operatorname{var}(h) \\
& \leq\left[\frac{2 N(f)}{\delta(f)}+\operatorname{var}\left(\frac{1}{\left|f^{\prime}\right|}\right)\right]\left(\|h\|_{1}+\operatorname{var}(h)\right)+\frac{1}{\delta(f)} \operatorname{var}(h) \\
& \leq\left[\frac{2 N(f)}{\delta(f)}+\operatorname{var}\left(\frac{1}{\left|f^{\prime}\right|}\right)+\frac{1}{\delta(f)}\right] \operatorname{var}(h)+\left[\frac{2 N(f)}{\delta(f)}+\operatorname{var}\left(\frac{1}{\left|f^{\prime}\right|}\right)\right]\|h\|_{1} .
\end{aligned}
$$

The above inequality trivially implies

$$
\begin{aligned}
\operatorname{var}\left(L_{f} h\right) \leq 6\left(\frac{N(f)}{\delta(f)} \vee 1\right)\left(\operatorname{var}\left(1 /\left|f^{\prime}\right|\right)\right. & \vee 1)\left(\frac{1}{\delta(f)} \vee 1\right) \cdot \operatorname{var}(h) \\
+ & 4\left(\frac{N(f)}{\delta(f)} \vee 1\right)\left(\operatorname{var}\left(1 /\left|f^{\prime}\right|\right) \vee 1\right) \cdot\|h\|_{1},
\end{aligned}
$$

where $x \vee y=\max (x, y)$.

An easy induction gives, after some simplifications,

Corollary 1.3. Let $F=f_{1} \circ \cdots \circ f_{n}$ be a composition of Lasota-Yorke maps. Then 


$$
\operatorname{var}\left(L_{F} h\right) \leq n 6^{n} \prod_{k=1}^{n}\left(\frac{N\left(f_{k}\right)}{\delta\left(f_{k}\right)} \vee 1\right)\left(\operatorname{var}\left(1 /\left|f_{k}^{\prime}\right|\right) \vee 1\right)\left(\frac{1}{\delta\left(f_{k}\right)} \vee 1\right) \cdot\left(\operatorname{var}(h)+\|h\|_{1}\right)
$$

1.2. Random bound. We are going to deduce from the previous Lasota-Yorke type inequalities the following fact.

Proposition 1.4. Let $f$ be an admissible random Lasota-Yorke map. Then there exists a $\mathbb{P}$-almost-everywhere finite function $M$ on $\Omega$ such that, and for all $h \in$ $L^{1}(m)$ with bounded variation, for all $\omega \in \Omega$,

$$
\operatorname{var}\left(L_{f\left(T^{-1} \omega\right) \circ \cdots \circ f\left(T^{-n} \omega\right)} h\right) \leq M(\omega)\left(\operatorname{var}(h)+\|h\|_{1}\right) \quad \forall n=1,2, \ldots
$$

and

$$
\limsup _{n \rightarrow \infty} \operatorname{var}\left(L_{f\left(T^{-1} \omega\right) \circ \cdots \circ f\left(T^{-n} \omega\right)} h\right) \leq M(\omega)\|h\|_{1} .
$$

Proof. Define $\sigma, \lambda$ and $\epsilon$ according to

$$
\begin{aligned}
0 \leq \sigma=\int_{\Omega} \log ^{+} \frac{1}{\delta}+\log ^{+} \frac{N}{\delta}+\log ^{+} \operatorname{var}\left(1 /\left|f_{\omega}^{\prime}\right|\right) d \mathbb{P}<\infty, \\
0<\lambda<\lim _{K \rightarrow \infty} \int_{\Omega} \log \delta \wedge K d \mathbb{P} \\
0<\epsilon<\frac{\lambda}{2(\sigma+\lambda+3+\log 12)} .
\end{aligned}
$$

Write $K(\omega)=\left(\frac{N(\omega)}{\delta(\omega)} \vee 1\right)\left(\operatorname{var}\left(1 /\left|f_{\omega}^{\prime}\right|\right) \vee 1\right)\left(\delta(\omega)^{-1} \vee 1\right)$. Let $R$ be so large that the following properties hold for all $\omega$ in a subset $\Omega_{1}$ of $\mathbb{P}$-measure greater than $1-\epsilon / 2$ :

$$
\begin{gathered}
\frac{1}{R} \log \delta(\omega) \cdots \delta\left(T^{R-1} \omega\right) \geq \lambda-\epsilon / 2, \\
\frac{1}{R} \log K(\omega) \cdots K\left(T^{R-1} \omega\right) \in[\sigma-\epsilon, \sigma+\epsilon] .
\end{gathered}
$$

We also assume that $R \leq 2^{R}, e^{\epsilon R / 2} \geq 3$.

Fix $\beta_{*}<\infty$ so large that we may assume that $\beta(f) \leq \beta_{*}$ for $\omega \in \Omega_{1}$ by reducing $\Omega_{1}$ by a measure at most $\epsilon / 2$. Hence $\mathbb{P}\left(\Omega_{1}\right)>1-\epsilon$.

Deleting a null set in $\Omega$, we may assume that for all $\omega \in \Omega$ and for all large $n$

$$
\begin{gathered}
\frac{1}{n} \#\left\{k=1, \ldots, n: T^{-k R+j} \omega \notin \Omega_{1}\right\}<\epsilon, \\
\frac{1}{n R} \sum_{k=1}^{n R} \log K\left(T^{-k} \omega\right) \in[\sigma-\epsilon, \sigma+\epsilon],
\end{gathered}
$$

where $0 \leq j=j(\omega)<R$ (note that $T$ but not $T^{R}$ is assumed to be ergodic). We set $j=0$, as this only introduces a finite factor (depending on $\omega$ ) in the desired bounds (1.5) and (1.6) (this follows from Lemma 1.2).

Let $\omega \in \Omega$ and $n \geq 1$. According to Lemmas 1.1 and 1.2 , we have

$$
\operatorname{var}\left(L_{\omega} h\right) \leq \alpha(\omega) \operatorname{var}(h)+\beta(\omega)\|h\|_{1}
$$

if we set $\alpha(\omega)=e^{-(\lambda-\epsilon) R} \geq 3 e^{-(\lambda-\epsilon / 2) R}$ and $\beta(\omega)=\beta_{*}$ for $\omega \in \Omega_{1}$, and $\alpha(\omega)=$ $\beta(\omega)=12^{R} \prod_{i=0}^{R-1} K\left(T^{i} \omega\right)$ otherwise. 
Let $h:[0,1] \rightarrow \mathbb{R}$ be an integrable function of bounded variation (which we think of as a density on $\left.T^{-n R} \omega \times[0,1]\right)$. Set $v_{0}=\operatorname{var}(h)$ and

$$
v_{k+1}=\alpha\left(T^{-(n-k) R} \omega\right) v_{k}+\beta\left(T^{-(n-k) R} \omega\right)\|h\|_{1} \quad \text { for } k=0,1, \ldots, n-1 .
$$

We have $\operatorname{var}\left(L_{f\left(T^{-(n-k) R-1} \omega\right) \circ \cdots \circ f\left(T^{-n R} \omega\right)} h\right) \leq v_{k}$. By induction we get from the definition of the $v_{k}$ 's that

$$
\begin{aligned}
v_{k+1}=\alpha & \left(T^{-n R} \omega\right) \cdots \alpha\left(T^{-(n-k) R} \omega\right) \operatorname{var}(h) \\
& +\sum_{j=0}^{k} \beta\left(T^{-(n-j) R} \omega\right) \cdot \alpha\left(T^{-(n-j-1) R} \omega\right) \cdots \alpha\left(T^{-(n-k) R} \omega\right) \cdot\|h\|_{1} .
\end{aligned}
$$

We compute (using (1.8) and (1.9))

$$
\begin{aligned}
\prod_{\substack{1 \leq k \leq n \\
T^{-k R} \omega \notin \Omega_{1}}} \alpha\left(T^{-k R} \omega\right) & =\prod_{1 \leq k \leq n} 12^{R} K\left(T^{-k R} \omega\right) \ldots K\left(T^{-k R+R-1} \omega\right) \\
& / \prod_{\substack{1 \leq k \leq n \\
T^{-k R} \omega \in \Omega_{1}}} 12^{R} K\left(T^{-k R} \omega\right) \ldots K\left(T^{-k R+R-1} \omega\right) \\
& \leq 12^{n R} e^{(\sigma+\epsilon) n R} /\left[12^{R} e^{(\sigma-\epsilon) R}\right]^{(1-\epsilon) n} \\
& \leq e^{\epsilon(\sigma+2+\log 12) n R} .
\end{aligned}
$$

Hence

$$
\begin{aligned}
\alpha\left(T^{-n R} \omega\right) \cdots \alpha\left(T^{-R} \omega\right) & \leq e^{-(\lambda-\epsilon)(1-\epsilon) n R} e^{\epsilon(\sigma+2+\log 12) n R} \\
& \leq e^{-(\lambda-\epsilon(\sigma+\lambda+\log 12+3)) n R} .
\end{aligned}
$$

This holds for $n$ large enough. Equivalently, for all $n \geq 0$,

$$
\alpha\left(T^{-n R} \omega\right) \cdots \alpha\left(T^{-R} \omega\right) \leq C_{1}(\omega) e^{-(\lambda-\epsilon(\sigma+\lambda+\log 12+3)) n R}
$$

for some measurable and $\mathbb{P}$-almost-everywhere finite function $C_{1}$. Remark that $\lambda-\epsilon(\sigma+\lambda+\log 12+3)>\lambda / 2>\epsilon$, because of (1.7).

On the other hand, the function $\log K$ is $\mathbb{P}$-integrable. Therefore, the convergence in the Birkhoff ergodic theorem implies that $\lim _{n \rightarrow \infty} \frac{1}{n} \log K\left(T^{-n} \omega\right)=0$ a.e. Hence, there exists a measurable and $\mathbb{P}$-almost-everywhere finite function $C_{2}$ such that

$$
\beta\left(T^{-k R} \omega\right) \leq C_{2}(\omega) e^{\epsilon k} \quad \forall \omega \in \Omega \text { and } \forall k=0,1,2, \ldots
$$

(1.10) gives

$$
\begin{aligned}
& \operatorname{var}\left(L_{f\left(T^{-1} \omega\right) \circ \cdots \circ f\left(T^{-n R} \omega\right)} h\right) \leq v_{n} \\
& \quad \leq C_{1}(\omega) e^{-\frac{\lambda}{2} n R} \operatorname{var}(h)+\sum_{j=0}^{n-1} C_{2}(\omega) e^{(n-j) \epsilon} \cdot C_{1}(\omega) e^{-\frac{\lambda}{2}(n-j-1) R} \cdot\|h\|_{1} .
\end{aligned}
$$

Proposition 1.4 is now obvious. 


\section{EXISTENCE OF AN A.C.I.M. FOR THE SKEW-PRODUCT}

This section is devoted to the construction of one $F$-invariant $\mathbb{P} \times m$-density. Obviously, $h$ is an invariant density iff it is a fixed point for the transfer operator $\mathcal{L}$. Therefore we are going to build an invariant density by trying to take some weak limit of $\frac{1}{n} \sum_{k=0}^{n-1} \mathcal{L}^{k} 1$.

We shall use the following "fibered" Mean Ergodic Theorem:

Proposition 2.1. Let $(\Omega, \mathbb{P})$ and $(I, m)$ be two probability spaces and let $T$ be an automorphism of $(\Omega, \mathbb{P})$. Let $\mathcal{L}$ be a positive contraction on $L^{1}(\mathbb{P} \times m)$ (i.e., $\mathcal{L}$ maps nonnegative functions to nonnegative functions and $\|\mathcal{L}\| \leq 1$ ) with the following properties:

1. For some measurable $M: \Omega \rightarrow\left[0, \infty\left[\right.\right.$, for all $h$ in some subset $\mathcal{H} \subset L^{\infty}(\mathbb{P} \times m)$ dense in $L^{1}(\mathbb{P} \times m)$, there exists $C(h)<\infty$ such that for all $\omega \in \Omega$ and all $n=1,2, \ldots$

$$
\left\|\left(\mathcal{L}^{n} h\right)(\omega, \cdot)\right\|_{\infty} \leq C(h) M(\omega),
$$

2. For all $\omega \in \Omega$ we have $m((\mathcal{L} h)(\omega, \cdot))=m\left(h\left(T^{-1} \omega, \cdot\right)\right)$ for all $h \in L^{1}(\mathbb{P} \times m)$. Then the averages $\frac{1}{n} \sum_{k=0}^{n-1} \mathcal{L}^{k}$ converge in $L^{1}(\mathbb{P} \times m)$ to $Q$, the projection on the fixed space of $\mathcal{L}$ with kernel the closure of $(\operatorname{Id}-\mathcal{L}) L^{1}(\mathbb{P} \times m)$.

If $\|M\|_{\infty}<\infty$, condition (1) is simply that the $\mathcal{L}^{n} h$ are bounded uniformly in $n$, and this proposition reduces to the Mean Ergodic Theorem [10, VIII.5.5]. But, because of the lack of uniformity in our assumptions, even $\mathcal{L}$ by itself is not a bounded operator on $L^{\infty}(\mathbb{P} \times m)$; this extension is therefore necessary.

Condition (2) means that $m$ is preserved by $\mathcal{L}$ fiberwise above $T$. Observe that this condition, together with positivity and the $T$-invariance of $\mathbb{P}$, implies that $Q$ is an isometry on positive $L^{1}$-functions. Hence

Corollary 2.2. Let $\mathcal{L}$ be as above. Then there exists $h \in L^{1}(\mathbb{P} \times m)$ with $h \geq 0$ and $\|h\|_{1}=1$ such that $\mathcal{L} h=h$.

Before proving this, we apply it to our problem:

Construction of an a.c.i.m. Proposition 1.4 shows that condition (1) is satisfied by our transfer operator $\mathcal{L}$ on $L^{1}(\mathbb{P} \times m)$ with

$\mathcal{H}$ is the set of $h \in L^{\infty}(\mathbb{P} \times m)$ such that the variation on fibers is uniformly bounded (i.e., $\sup _{\omega} \operatorname{var}(h(\omega, \cdot))<\infty$ ).

We may set $C(h)=2\|h\|_{\infty}+\sup _{\omega} \operatorname{var}(h(\omega, \cdot))$ (assuming $M(\omega) \geq 1$ for all $\omega$ ). To approximate a function in $L^{1}$ by an element of $\mathcal{H}$, we use conditional expectation with respect to a generating sequence of finer and finer finite partitions into subsets of the form $A \times I$, where $A \subset \Omega$ is Borel and $I$ is an interval. Condition (2), the invariance of Lebesgue measure under each one-dimensional transfer operator $L_{\omega}$, is clear. Thus Proposition 2.1 applies and its corollary gives the first point of our Main Theorem: the existence of an a.c.i.m. for $F$.

We turn to the proof of the proposition. To take care of the unboundedness of $M$, we renormalize and work with a new reference measure, $\lambda=M \cdot \mathbb{P} \times m . \lambda$ is a $\sigma$-finite positive measure. It is not necessarily finite, forcing us to clear up some things with the next lemma. 
Recall that a set of measures $\mu$ on $\Omega \times I$ is $\lambda$-tight if for every $\epsilon>0$ there exists $X_{\epsilon}$ with finite $\lambda$-measure such that $|\mu|\left(\Omega \times I \backslash X_{\epsilon}\right)<\epsilon$ for every measure $\mu$ in the set.

Lemma 2.3. Let $K$ be a bounded subset of $L^{\infty}(\lambda)$ such that the collection of measures $h \cdot \lambda, h \in K$, is $\lambda$-tight.

Then $K$ is a conditionally weakly compact subset of $L^{1}(\lambda)$, i.e., it has compact closure in the topology defined by the functionals

$$
h \mapsto \int h g d \lambda \quad\left(g \in L^{\infty}(\lambda)\right)
$$

(remember that $L^{\infty}(\lambda)$ is the dual of $L^{1}(\lambda)$ ).

Proof of the lemma. By the Banach-Alaoglu theorem, the unit ball of $L^{\infty}(\lambda)$ is compact in the weak star topology defined by the functionals: $h \mapsto \int h g d \lambda$, where $g$ ranges over $L^{1}(\lambda)$. This implies the statement of the lemma, but restricted to $g \in L^{\infty}(\lambda)$ such that $\lambda(g \neq 0)<\infty$. We extend it to general $g$. It is enough to prove the following for any sequence $h_{1}, h_{2}, \ldots$ of positive or negative parts of elements of $K$ : if this sequence converges in the weak star topology to some limit $h \in L^{\infty}(\lambda)$, then, for each non-negative $g \in L^{\infty}(\lambda), \lim _{n \rightarrow \infty} \int h_{n} g d \lambda=\int h g d \lambda$.

We first consider the case where $g=1_{E}$ for some measurable $E \subset \Omega \times I$. Write $E$ as an increasing union of subsets $E_{1}, E_{2}, \ldots$, each one with finite $\lambda$-measure. We have $\lambda(E)=\sup _{m} \lambda\left(E_{m}\right)$, and the weak star convergence gives, for each $m \geq 1$, $(h \cdot \lambda)\left(E_{m}\right)=\lim _{n \rightarrow \infty}\left(h_{n} \cdot \lambda\right)\left(E_{m}\right) \leq \liminf _{n \rightarrow \infty}\left(h_{n} \cdot \lambda\right)(E)$. Hence

$$
(h \cdot \lambda)(E) \leq \liminf _{n \rightarrow \infty}\left(h_{n} \cdot \lambda\right)(E) \quad \text { for all measurable } E .
$$

Assume that for some subset $E_{0}$ this is a strict inequality. Let $\epsilon$ be the "loss" in measure, i.e., $\epsilon=\liminf _{n \rightarrow \infty}\left(h_{n} \cdot \lambda\right)\left(E_{0}\right)-(h \cdot \lambda)\left(E_{0}\right)>0$. Observe that tightness of the $h_{n} \cdot \lambda$ together with $L^{\infty}$-boundedness of $h_{1}, h_{2}, \ldots$ implies that $\sup _{n}\left(h_{n} \cdot \lambda\right)(\Omega \times I)<\infty$, so that $0<\epsilon<\infty$. In particular, by writing $\Omega \times I=$ $E_{0} \cup\left(\Omega \times I \backslash E_{0}\right)$ and applying (2.4) to $\Omega \times I \backslash E_{0}$, we see that $\Omega \times I$ must exhibit at least the same loss $\epsilon$; hence we may take $E_{0}=\Omega \times I$.

By the tightness hypothesis again, there exists a subset $Z$ of finite $\lambda$-measure such that $\left(h_{n} \cdot \lambda\right)(\Omega \times I \backslash Z) \leq \epsilon / 2$ for all $n=1,2, \ldots$ Now

$$
\liminf _{n \rightarrow \infty}\left(h_{n} \cdot \lambda\right)(\Omega \times I) \leq(h \cdot \lambda)(Z)+\epsilon / 2 \leq(h \cdot \lambda)(\Omega \times I)+\epsilon / 2 .
$$

This contradicts the definition of $0<\epsilon<\infty$. Hence (2.4) is an equality.

Now consider an arbitrary non-negative $g \in L^{\infty}(\lambda)$. Let $\epsilon>0$. By tightness, there exists $Y$ with $\lambda$-finite measure such that $\left(h_{n} \cdot \lambda\right)(\Omega \times I \backslash Y) \leq \epsilon / 3\|g\|_{\infty}$ for all $n$ (implying, by (2.4), that the same inequality holds for the limit, $h$ ). Now $g 1_{Y}$ is in $L^{1}(\lambda)$; hence, for large $n,\left|\int h_{n} g 1_{Y} d \lambda-\int h g 1_{Y} d \lambda\right| \leq \epsilon / 3$. Therefore

$$
\begin{aligned}
\left|\int h_{n} g d \lambda-\int h g d \lambda\right| & \leq\left|\int h_{n} g\left(1-1_{Y}\right)+\left(h_{n}-h\right) g 1_{Y}+h g\left(1_{Y}-1\right) d \lambda\right| \\
& \leq\|g\|_{\infty}\left(h_{n} \cdot \lambda\right)(\Omega \times I \backslash Y)+\epsilon / 3 \\
& \quad+\|g\|_{\infty}(h \cdot \lambda)(\Omega \times I \backslash Y) \\
& \leq \epsilon \quad
\end{aligned}
$$

for all large $n$. As $\epsilon>0$ was arbitrarily small, the weak convergence and hence the lemma are proved. 
Proof of the proposition. Let $h \in \mathcal{H}$. We set

$$
h_{n}=\frac{1}{M}\left(\frac{1}{n} \sum_{k=0}^{n-1} \mathcal{L}^{k} h\right), \quad n=1,2, \ldots
$$

The normalization by $M$ ensures that $\sup _{n \geq 1}\left\|h_{n}\right\|_{\infty}<\infty$ by condition (1). Using condition (2), each $h_{n} \cdot \lambda$ projects onto $\Omega$ to a measure dominated by $\|h\|_{\infty} \cdot \mathbb{P}$. Indeed, for any measurable $E \subset \Omega$

$$
\begin{aligned}
\left|\left(h_{n} \cdot \lambda\right)(E \times I)\right| & \leq \frac{1}{n} \sum_{k=0}^{n-1} \int_{E \times I} \frac{1}{M}\left\|\mathcal{L}^{k} h(\omega, \cdot)\right\|_{1} d \lambda=\frac{1}{n} \sum_{k=0}^{n-1} \int_{E}\left\|\mathcal{L}^{k} h(\omega, \cdot)\right\|_{1} d \mathbb{P} \\
& =\frac{1}{n} \sum_{k=0}^{n-1} \int_{E}\left\|h\left(T^{-k} \omega, \cdot\right)\right\|_{1} d \mathbb{P} \leq\|h\|_{\infty} \mathbb{P}(E) .
\end{aligned}
$$

Therefore the sequence $h_{1} \cdot \lambda, h_{2} \cdot \lambda, \ldots$ is $\lambda$-tight and we may apply the previous lemma. Now the weak $L^{1}(\lambda)$-convergence of $h_{n}$ is the same as the weak $L^{1}(\mathbb{P} \times m)$ convergence of $M \cdot h_{n}$. Therefore the lemma shows that

$$
\left\{\frac{1}{n}\left(\sum_{k=0}^{n-1} \mathcal{L}^{k} h\right)\right\}_{n=1,2, \ldots} \text { is } L^{1}(\mathbb{P} \times m) \text {-conditionally weakly compact. }
$$

This holds for all $h \in \mathcal{H}$. $\mathcal{H}$ is dense in $L^{1}(\mathbb{P} \times m)$. Therefore, by [10, VIII.5.3] we have that the operators $\frac{1}{n} \sum_{k=0}^{n-1} \mathcal{L}^{k}$ converge in $L^{1}$ to the projection $Q$ with range the set of fixed points of $\mathcal{L}$ and kernel the closure of $(\operatorname{Id}-\mathcal{L}) L^{1}(\mathbb{P} \times m)$, by [10] VIII.5.2].

This finishes the proof of the proposition.

\section{Bound on the multiplicity of the A.C.I.M.}

To prove the explicit bound (0.4), we first remark that the support of any a.c.i.m. contains intervals by establishing the regularity of the invariant densities. From this we deduce that assumption (A2) implies that there can be only a finite number of mutually singular a.c.i.m.'s. This immediately implies that there exist finitely many ergodic a.c.i.m.'s such that any a.c.i.m. is a convex combination of those: the set of a.c.i.m.'s is, as claimed in our Main Theorem, a finite-dimensional simplex.

We also remark that simple finiteness can be proved more abstractly, by a compactness argument-we learnt this from G. Keller in the deterministic case.

3.1. Regularity of arbitrary a.c.i.m.'s. We first prove that the densities of the a.c.i.m.'s constructed as above have finite variation on fibers:

Lemma 3.1. Let $h \in \mathcal{H}$. For $\mu=\lim _{n \rightarrow \infty} \frac{1}{n} \sum_{k=0}^{n-1} \mathcal{L}^{k} h \cdot \mathbb{P} \times m$, we have

$$
\operatorname{var}\left(\frac{d \mu}{d \mathbb{P} \times m}(\omega, \cdot)\right) \leq M(\omega) \sup _{\alpha}\left\|h_{\alpha}\right\|_{1}<\infty \quad \text { for } \mathbb{P} \text {-a.e. } \omega
$$

(after modification on a null set).

Proof. First note that the convergence in $L^{1}(\mathbb{P} \times m)$ of $\left(\frac{1}{n} \sum_{k=0}^{n-1} \mathcal{L}^{k} h\right)_{n \geq 1}$ implies that there exist $n_{1}<n_{2}<\cdots$ such that $\left(\frac{1}{n_{i}} \sum_{k=0}^{n_{i}-1} \mathcal{L}^{k} h\right)(\omega, \cdot)$ converges in $L^{1}(m)$ to $\frac{d \mu}{d \mathbb{P} \times m}(\omega, \cdot)$ for $\mathbb{P}$-almost every $\omega$. 
Recall (1.6): $\limsup _{n \rightarrow \infty} \operatorname{var}\left(\left(\mathcal{L}^{n} h\right)(\omega, \cdot)\right) \leq M(\omega) \sup _{\alpha}\left\|h_{\alpha}\right\|_{1} \leq M(\omega)\|h\|_{\infty}<$ $\infty$, independently of the finite variation on fibers of $h$. But, $\left\{g \in L^{1}(m):\|g\|_{1} \leq\right.$ $\|h\|_{\infty}$ and $\left.\operatorname{var}(g) \leq M(\omega) \sup _{\alpha}\left\|h_{\alpha}\right\|_{1}+\epsilon\right\}$ is a compact subset of $L^{1}(m)$, for each $\epsilon>0$. Hence $\frac{d \mu}{d \mathbb{P} \times m}(\omega, \cdot)$ must belong to it. But this is the statement of the lemma.

We extend this bounded variation property to the general case:

Proposition 3.2. Let $\nu$ be an invariant probability measure such that $\nu \ll \mathbb{P} \times m$. Let $M: \Omega \rightarrow[1, \infty[$ be as in (1.6). Then

$$
\operatorname{var}\left(\frac{d \nu}{d \mathbb{P} \times m}(\omega, \cdot)\right) \leq M(\omega)<\infty \quad \text { for } \mathbb{P} \text {-a.e. } \omega
$$

(after modification on a null set).

Proof. We write $\nu$ as $h \cdot \mathbb{P} \times m$ with $h \in L^{1}(\mathbb{P} \times m), h \geq 0, \int_{[0,1]} h_{\omega} d m=1$ for $\mathbb{P}$-a.a. $\omega$ and with $\mathcal{L} h=h$. Using the density of $\mathcal{H}$ in $L^{1}(\mathbb{P} \times m)$, we first find $h^{\epsilon} \in \mathcal{H}$ such that $\left\|h-h^{\epsilon}\right\|_{1} \leq \epsilon$. We apply the preceding section to it and see that $\frac{1}{n} \sum_{k=0}^{n-1} \mathcal{L}^{k} h^{\epsilon}$ converges in $L^{1}$ to an invariant density $g^{\epsilon}$. As $\|\mathcal{L}\|_{1}=1$,

$$
\left\|h-\frac{1}{n} \sum_{k=0}^{n-1} \mathcal{L}^{k} h^{\epsilon}\right\|_{1} \leq\left\|h-h^{\epsilon}\right\|_{1} \leq \epsilon .
$$

The inequality goes to the limit $n \rightarrow \infty:\left\|h-g^{\epsilon}\right\|_{1} \leq \epsilon$. That is, $g^{\epsilon}$ goes to $h$ as $\epsilon$ goes to zero.

But $\operatorname{var}\left(g^{\epsilon}(\omega, \cdot)\right) \leq M(\omega)$ for every $\omega \in \Omega$. From the $L^{1}$-compactness of $\{g \in$ $\left.L^{1}(m): \operatorname{var}(g) \vee\|g\|_{1} \leq K\right\}$ for finite $K$, we get that $\operatorname{var}(h(\omega, \cdot))$ must be finite $\mathbb{P}$-a.e.

Bound on the multiplicity. Assume that there exist $r$ mutually singular a.c.i.m.'s $\mu_{1}, \ldots, \mu_{r}$. In particular there exist invariant and pairwise disjoint measurable subsets $E_{1}, \ldots, E_{r} \subset \Omega \times[0,1]$ such that $\mu_{i}\left(E_{i}\right)=1$.

It is obvious that any non-negative function of bounded variation which has positive Lebesgue integral admits a non-trivial interval on which it is positive. Hence, for $\mathbb{P}$-a.a. $\omega$, there exist non-trivial intervals $I_{1}, \ldots, I_{r}$ such that $F^{n}\left(\omega \times I_{i}\right) \subset$ $E_{i} \cap\left(T^{n} \omega \times[0,1]\right)$ modulo $m$, for all $n=0,1, \ldots$ We fix such an $\omega$.

Set $I_{i}(0)=I_{i}$ and, for $n \geq 0, I_{i}(n+1)=f_{T^{n} \omega} I_{i}(n) \cap J$, where $J$ is the open interval of $f_{T^{n} \omega}$ which maximizes the length of $I_{i}(n+1)$. Recall that the intervals of a Lasota-Yorke map are simply the largest intervals on which the map is continuous and monotonic.

Define $N_{i}(n)$ to be the number of intervals of $f_{T^{n} \omega}$ meeting $I_{i}(n)$. Obviously,

$$
m\left(I_{i}(n+1)\right) \geq \frac{\delta\left(T^{n} \omega\right)}{N_{i}(n)} m\left(I_{i}(n)\right) .
$$

As $m\left(I_{i}(n)\right) \leq 1$ in all cases, we get

$$
\sum_{k=0}^{n-1} \log \frac{N_{i}(k)}{\delta\left(T^{k} \omega\right)} \geq \log m\left(I_{i}(0)\right) \quad \forall n=1,2, \ldots
$$


$N_{i}(k)-1$ is the number of singularities of $f_{T^{k} \omega}$ contained in the open interval $I_{i}(k)$. Hence, $I_{1}(k), \ldots, I_{r}(k)$ being disjoint, $N_{1}(k)-1+\cdots+N_{r}(k)-1 \leq N\left(T^{k} \omega\right)-$ 1. Thus

$$
N_{1}(k) \cdots N_{r}(k) \leq\left(\frac{N\left(T^{k} \omega\right)+r-1}{r}\right)^{r} .
$$

Hence, summing (3.3) over $i=1, \ldots, r$ and dividing by $r$, we get

$$
\sum_{k=0}^{n-1} \log \left(\frac{N\left(T^{k} \omega\right)+r-1}{r \delta\left(T^{k} \omega\right)}\right) \geq \frac{1}{r} \sum_{i=1}^{r} \log m\left(I_{i}(0)\right) .
$$

Thus

$$
\frac{1}{n} \sum_{k=0}^{n-1} \log \frac{1+\left(N\left(T^{k} \omega\right)-1\right) / r}{\delta\left(T^{k} \omega\right)} \geq \frac{1}{n} \text { const. }
$$

Now $(1 / r) N \leq 1+(N-1) / r \leq(1+1 / r) N$, as $N \geq 1$ and $r \geq 1$. Hence, $\log \frac{1+(N-1) / r}{\delta}-\log N / \delta$ is a bounded function. But $\log N / \delta$ is integrable by assumption (A2) and the fact that $N / \delta \geq 1$ (otherwise the image of some interval of the map would not fit into [0,1]). Thus we may let $n \rightarrow \infty$ and apply the ergodic theorem, to find that

$$
\int_{\Omega} \log \frac{1+(N(\omega)-1) / r}{\delta(\omega)} d \mathbb{P} \geq 0
$$

The integrand above is a non-increasing function of $r$. It is integrable for $r=1$. Hence, by the monotone convergence theorem, the integral decreases to $\int_{\Omega} \log \frac{1}{\delta} d \mathbb{P}$, which is strictly negative, when $r \rightarrow \infty$. Thus the above inequality gives a (nontrivial) bound on $r$, and in particular we obtain finiteness.

Abstract proof of finiteness. The above explicit bound uses the "geometry" of the interval. We now give an abstract and simple proof of the finiteness (without any bound), suitable for generalization to systems only assumed to satisfy "good LasotaYorke inequalities" (see 7]). We learnt this argument (applied to the deterministic case) from G. Keller.

Assume that there are infinitely many a.c.i.m.'s for the skew-product. Choose a countable subset $h_{1}, h_{2}, \ldots$ of invariant, normalized, true functions which are pairwise distinct in $L^{1}(\mathbb{P} \times m)$. Consider the vector space $\mathcal{D}$ generated by these, i.e., the set of all finite linear combination of $h_{n}$ 's. For each $\omega \in \Omega$, the restriction to $\omega \times[0,1]$ defines a linear map $h \mapsto h_{\omega}$. This map is injective for a.a. $\omega$. Indeed, consider for instance the set of $\omega$ 's which are typical with respect to the countably many subsets $\left\{\omega \in \Omega:\left\|\alpha_{1} h_{1}+\cdots+\left.\alpha_{n} h_{n}\right|_{\omega}\right\|_{1} \leq r_{n}\right\}$ for all $n=1,2, \ldots$ and all rationals $\alpha_{1}, \ldots, \alpha_{n}, r_{n}$. For such an $\omega$, for any $h \in \mathcal{D}, \frac{1}{n} \sum_{k=0}^{n-1}\left\|h_{T^{k}}\right\|_{1} \stackrel{n \rightarrow \infty}{\rightarrow}\|h\|_{1}$, which is not zero provided $h \neq 0$. But $\left\|h_{T^{k}{ }^{\omega}}\right\|_{1}=\left\|L_{\omega}^{k} h_{\omega}\right\|_{1} \leq\left\|h_{\omega}\right\|_{1}$. Hence $h_{\omega} \neq 0$ for $h \neq 0$, proving that $h \mapsto h_{\omega}$ is one-to-one.

Thus the image $\mathcal{D}_{\omega}$ of $\mathcal{D}$ must also be infinite-dimensional for a.a. $\omega$.

Now Proposition 1.4 implies that, for a.a. $\omega$, the unit ball of $\mathcal{D}_{\omega} \subset L^{1}(m)$ is made of functions with uniformly bounded variation. Hence this unit ball is relatively compact as a subset of $L^{1}(m)$. But this means that the space $\mathcal{D}_{\omega}$ is finite-dimensional. The contradiction proves the finiteness. 


\section{S.R.B. MEASURES FOR THE RANDOM MAP}

We prove Theorem 0.5 , restated as:

Proposition 4.1. Let $\mu_{1}, \ldots, \mu_{r}$ be the finite collection of all ergodic a.c.i.m.'s for the skew-product $F$ on $\Omega \times[0,1]$. Define $\nu_{i}$ as the projection on $[0,1]$ of the a.c.i.m. $\mu_{i}$. Then each $\nu_{i}$ is an absolutely continuous S.R.B. measure. Moreover, $\mathbb{P}$-almost surely, the union of their basins has total Lebesgue measure.

Proof. The Birkhoff Ergodic Theorem says that the set $B_{i} \subset \Omega \times[0,1]$ of points $(\omega, x)$ such that $\frac{1}{n} \sum_{k=0}^{n-1} \delta_{F^{k}(\omega, x)}$ converges vaguely to $\mu_{i}$ has $\mu_{i}$-measure 1 (we endow the Lesbesgue space $\Omega$ with the topology defined by some identification of it with $([0,1], m))$. Projecting this to $[0,1]$, we get the defining property of an S.R.B. measure provided that we check that $B_{\omega}\left(\nu_{i}\right) \stackrel{\text { def }}{=} B_{i} \cap(\omega \times[0,1])$ has positive Lebesgue measure for $\mathbb{P}$-almost all $\omega$. Now $\mathbb{P} \times m\left(B_{i}\right)>0$, as $\mu_{i}$ is an a.c.i.m. It is now enough to recall that $B_{i}$ is $F$-invariant and $(T, \mathbb{P})$ is ergodic, to see that $m\left(B_{\omega}\left(\nu_{i}\right)\right)>0, \mathbb{P}$-almost surely in $\omega$.

It remains to check that $\bigcup_{i=1}^{r} B_{\omega}\left(\nu_{i}\right)=[0,1](\bmod m), \mathbb{P}$-almost surely. We have to prove that the following set $E$ is a $\mathbb{P} \times m$-null set:

$$
E=\left\{(\omega, x) \in \Omega \times[0,1]: \forall i=1, \ldots, r \quad \frac{1}{n} \sum_{k=0}^{n-1} \delta_{F^{k}(\omega, x)} \nrightarrow \mu_{i}\right\} .
$$

Note that $F(E) \subset E$. Hence $\frac{1}{n} \sum_{k=0}^{n-1} \mathcal{L}^{k} 1_{E}$ is zero outside $E$ for each $n=1,2, \ldots$. Using $L^{1}$-convergence, this implies the same for the limit $Q 1_{E}$. Now, $\left\|Q 1_{E}\right\|_{1}=$ $\left\|1_{E}\right\|_{1}=\mathbb{P} \times m(E)$. Hence, if $\mathbb{P} \times m(E)>0, \frac{1}{\mathbb{P} \times m(E)} Q 1_{E} \cdot \mathbb{P} \times m$ should be an a.c.i.m., hence with ergodic components $\mu_{1}, \ldots, \mu_{r}$. In particular, applying the ergodic theorem, for $\mathbb{P} \times m$-almost all points of $\left\{Q 1_{E}>0\right\}$, the empirical measures should converge to one of the $\mu_{i}$ 's. This contradicts $\left\{Q 1_{E}>0\right\} \subset E$, unless $E$ is a null set.

\section{REFERENCES}

[1] V. Baladi, Correlation spectrum of quenched and annealed equilibrium states for random expanding maps, Commun. Math. Phys. 186 (1997), 671-700. MR 98i:58188

[2] V. Baladi, A. Kondah, B. Schmitt, Random correlations for small perturbations of expanding maps, Random Comput. Dynam. 4 (1996), 179-204. MR 97e:58139

[3] M. Blank, G. Keller, Stochastic stability versus localization in one-dimensional chaotic dynamical systems, Nonlinearity 10 (1997), 81-107. MR 98a:58101

[4] T. Bogenschütz, V.M. Gundlach, Symbolic dynamics for expanding random dynamical systems, Random Comput. Dynamics 1 (1992/93), 219-227. MR 93j:58042

[5] T. Bogenschütz, V.M. Gundlach, Ruelle's transfer operator for random subshifts of finite type, Ergod. Th. \& Dynam. Sys. 15 (1995), 413-447. MR 96m:58133

[6] J. Buzzi, Absolutely continuous invariant measures for generic multi-dimensional piecewise expanding and affine maps, Int. J. Bifurcations \& Chaos 9 (1999), 1743-1750.

[7] Exponential decay of correlations for random Lasota-Yorke maps, (preprint I.M.L. 98-12), to appear Commun. Math. Phys.

[8] - A.c.i.m.'s for arbitrary expanding piecewise real-analytic mappings of the plane, (preprint I.M.L. 98-13), to appear Ergod. Th. \& Dynam. Sys.

[9] A.c.i.m.'s as equilibrium states for piecewise invertible dynamical systems, (preprint I.M.L. 98).

[10] N. Dunford, T. Schwartz, Linear operators. Part I. General theory, Wiley, New York, 1958. MR 22:8302

[11] P. Ferrero, B. Schmitt, Produits aléatoires d'opérateurs matrices de transfert, Probab. Th. Related Fields 79 (1988), 227-248. MR 90e:47006 
[12] P. Góra, A. Boyarsky, Absolutely continuous invariant measures for piecewise expanding $C^{2}$ transformations in $R^{N}$, Israel J. Math. 67 (1989), 272-286. MR 91c:58061

[13] P. Góra, B. Schmitt, Un example de transformation dilatante et $C^{1}$ par morceaux de l'intervalle sans probabilité absolument continue invariante, Ergod. Th. \& Dynam. Syst. 9 (1989), 101-113. MR 90h:58052

[14] K. Khanin, Y. Kifer, Thermodynamic formalism for random transformations and statistical mechanics, Sinai's Moscow Seminar on Dynamical Systems, A.M.S. Translation, Series 2, vol. 171, Amer. Math. Soc., Providence, Rhode Island, 1996, pp. 107-140. MR 46j:58136

[15] Yu. Kifer, Ergodic theory of random transformations, Birkhäuser, Boston, 1986. MR 89c:58069

[16] , Random perturbations of dynamical systems, Birkhäuser, Boston, 1988. MR 91e:58159

[17] A. Kondah, Les endomorphismes dilatants de l'intervalle et leurs perturbations aléatoires, Thèse de l'Université de Bourgogne, Dijon, 1991.

[18] U. Krengel, Ergodic theorems, de Gruyter, Berlin, 1985. MR 87i:28001

[19] A. Lasota, J.A. Yorke, On the existence of invariant measures for piecewise monotonic transformations, Trans. Amer. Math. Soc. 186 (1973), 481-488. MR 49:538

[20] T. Morita, Random iteration of one-dimensional transformations, Osaka J. Math. 22 (1985), 489-518. MR 87a:58100

[21] S. Pelikan, Invariant densities for random maps of the interval, Trans. Amer. Math. Soc. 281 (1984), 813-825. MR 85i:58070

[22] M. Rychlik, Bounded variation and invariant measures, Studia Math. 76 (1983), 69-80. MR 85h:28019

[23] M. Tsujii, A.c.i.m. for piecewise real-analytic expanding mappings of the plane (preprint, Hokkaido, 1998).

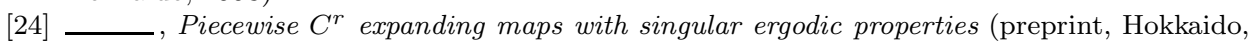
1998).

Institut de Mathématiques de Luminy, 163 aV. De Luminy, Case 907, 13288 Marseille Cedex 9, France

Current address: Centre de Mathématiques, Ecole Polytechnique, 91128 Palaiseau Cedex, France

E-mail address: buzzi@iml.univ-mrs.fr 